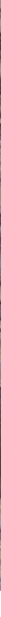

\title{
La conservación del vidrio de ventana romano: perspectivas de actuación tras su estudio arqueométrico
}

\author{
Almudena Velo Gala, Manuel García Heras
}

\begin{abstract}
Resumen: La arqueometría es una herramienta imprescindible en el estudio del vidrio antiguo, tanto desde una perspectiva arqueológica como en lo que respecta a la conservación de estos materiales. Para entender cómo afectan los procesos postdeposicionales al vidrio arqueológico y los tratamientos aplicados tras su descubrimiento, se seleccionó un conjunto de vidrios de ventana romanos procedentes de varios yacimientos de la Bética para su caracterización químico-física. Las muestras se observaron mediante microscopía electrónica de barrido de emisión de campo (MEBEC) y se microanalizaron con espectrometría de dispersión de energías de rayos X (EDS). Los resultados revelaron que muchos de los vidrios habían perdido las capas de degradación como consecuencia de los tratamientos de limpieza y los sistemas de almacenamiento. La importante información que aportan las superficies de estos vidrios respecto a las técnicas de elaboración e instalación de los paneles plantea la necesidad de incorporar nuevos protocolos de conservación tras su hallazgo.
\end{abstract}

Palabras clave: conservación, vidrio arqueológico, vidrio de ventana romano, arqueometría, procesos de alteración

\section{Roman window glass conservation: prospects for action after its archaeometric study}

Abstract: Archaeometry is an essential tool in the study of ancient glass, both from an archaeological perspective and with regard to conservation of these materials. To understand how postdepositional processes and the treatments applied affect archaeological glass after its discovery, a set of Roman window glasses from several archaeological sites from the Baetica Roman province were selected for chemical-physical characterization. Samples were observed through field emission scanning electron microscopy (FESEM) and microanalysed with energy dispersive X-ray spectrometry (EDS). The results indicated that many of the glasses had lost their degradation layers as a result of cleaning treatments and storage systems. The important information obtained from the surfaces of these glasses with respect to processing and installation techniques raises the need to incorporate new conservation protocols after their finding.

Keyword: conservation, archaeological glass, roman window glass, archaeometry, alteration processes

\section{A conservação do vidro de janela romana: perspetivas de ação após seu estudo arqueométrico}

Resumo: A arqueometria é uma ferramenta essencial no estudo do vidro antigo, tanto do ponto de vista arqueológico como no que diz respeito à conservação destes materiais. Para entender como os processos pós-deposicionais afetam o vidro arqueológico e os tratamentos aplicados após sua descoberta, um conjunto de vidros de janela romanos de vários locais da Baetica foi selecionado para sua caracterização físico-química. As amostras foram observadas por microscopia eletrónica de varrimento por emissão de campo (FESEM) e microanálise por espectrometria de raios $X$ dispersiva de energia (EDS). Os resultados revelaram que muitos dos vidros perderam as camadas de degradação como resultado de tratamentos de limpeza e sistemas de armazenamento. As importantes informações fornecidas pelas superfícies destes vidros, no que diz respeito às técnicas de preparação e instalação dos painéis, levanta a necessidade de incorporar novos protocolos de conservação após a sua descoberta.

Palavras-chave: conservação, vidro arqueológico, vidro de janela romana, arqueometria, processos de alteração 


\section{Estado de la cuestión y objetivos}

A pesar de que el vidrio fue un material destacado en la Antigüedad para la elaboración de todo tipo de objetos y recipientes, su escasa preservación y un deficiente interés como marcador cronológico en las intervenciones arqueológicas ha causado que los trabajos sobre vidrio sean menos numerosos en comparación con otros materiales. Sin embargo, la aplicación de la Arqueometría en su estudio ha supuesto en las últimas décadas un gran avance en la investigación de los restos de vidrio hallados en los yacimientos, tanto desde una perspectiva histórica como en el ámbito de la conservación.

En el campo de la Arqueología, las técnicas de análisis químico-físico han permitido investigar cuestiones relacionadas con la elaboración de los objetos, el posible origen del vidrio utilizado, su uso, consumo y comercio. Los resultados obtenidos han evidenciado la importancia del vidrio en el progreso económico de determinadas sociedades y en épocas concretas, impulsando la creación de un campo de investigación innovador y esencial para comprender la evolución de la industria del vidrio a lo largo de la Historia. Asimismo, estos análisis han servido para afrontar nuevos procedimientos de conservación-restauración del vidrio arqueológico, que facilitan la comprensión de los procesos de degradación de estos materiales en diferentes contextos medioambientales y la incidencia de los tratamientos aplicados tras su hallazgo.

En lo que respecta a la Península Ibérica, los estudios sobre vidrio arqueológico aún son escasos, destacando algunos trabajos en el ámbito de la conservación, tanto de temas generales (García-Heras y Villegas 2004) como centrados en vidrios romanos (Agua et al. 2015; Palomar et al. 2012 2013). A pesar de que muchas de estas publicaciones abordan conjuntos de vidrios de diversa tipología, existen algunos, como el vidrio de uso arquitectónico para cierre de vanos, que pasan desapercibidos por su desconocimiento $y$, solo en casos excepcionales, se procede a su investigación (Velo-Gala et al. 2019). No obstante, restos de paneles de vidrio aparecen con relativa frecuencia en las excavaciones, los cuales aportan una información relevante sobre la arquitectura, el comercio y la producción del vidrio del periodo al que se adscriben.

Son numerosos los factores que influyen en el estado de conservación del vidrio de ventana arqueológico en contextos de enterramiento: el uso de estos paneles durante su vida útil, los procesos postdeposicionales, la composición química y las técnicas de elaboración, así como los distintos tratamientos de conservación-restauración y almacenaje aplicados tras su recuperación.

Muchas de las patologías detectadas en el vidrio de ventana son consecuencia de la pérdida de funcionalidad de los edificios donde se instalaron. Tras el abandono de las estructuras algunos de ellos fueron sustraídos, completos o fragmentados intencionadamente, para su reciclado, lo que ha influido en el registro arqueológico de estos objetos (Velo Gala 2020). Entre los materiales recuperados también se observa un mayor número de fragmentos pertenecientes a los bordes, debido a que estas partes eran más difíciles de sustraer de los bastidores o paramentos, quedando en el entorno de los edificios tras su desplome o cerca del lugar donde se ubicaron las ventanas. Otros fueron arrojados como desechos secundarios a basureros, aunque su valor como objeto reciclable por el abundante material que aportaban, hace que estos hallazgos sean puntuales y escasos en estos contextos. Precisamente, a consecuencia de esta práctica es menos frecuente y excepcional la documentación de paneles completos o de un número abundante de fragmentos en un mismo yacimiento. En cualquiera de estas situaciones, los vidrios sufren una serie de daños principalmente físicos, ocasionando deformaciones en el material, grietas y fisuras, los cuales pueden continuar en el curso de su enterramiento debido a las presiones ejercidas por los estratos de cubrición (Davison 2003: 182).

Uno de los principales factores que determina la durabilidad química del vidrio es su composición, siendo los vidrios de silicato sódico cálcico característicos de época romana los que mayor estabilidad presentan en contextos arqueológicos (Freestone 2001: 619). A pesar de ello, la existencia de agua de forma prolongada durante el largo periodo de enterramiento es un factor determinante para el inicio de alteraciones químicas que, en algunos casos, llega a afectar a toda la pieza y producir su completa disgregación. El agua acaba adsorbiéndose en los grupos silanoles [ $\mathrm{E} \mathrm{Si}-\mathrm{OH}$ ] de la superficie del vidrio a través de enlaces de hidrógeno, provocando una apertura de la red vítrea y la entrada de moléculas de agua hacia capas más internas. Este proceso se conoce como ataque hidrolítico y es el responsable de la capa iridiscente formada por pequeñas y delgadas láminas de sílice hidratada que caracteriza a los vidrios arqueológicos (Cox y Ford 1993: 5641), también conocida como "capa de gel de sílice" (Davison 2003: 175). La superposición laminar produce una difracción de la luz cuando incide sobre la misma y genera el fenómeno de la iridiscencia, llegando a provocar cierta opacidad en vidrios incoloros (Davison 2003: 183). Además, su consistencia es débil y frecuentemente se desprende del vidrio cuando los objetos son manipulados.

La humedad también es un factor determinante para la actuación de las sales solubles e insolubles en los vidrios arqueológicos. En el caso de las sales insolubles forman depósitos superficiales tras su cristalización, mientras que cuando se trata de sales solubles pueden situarse en pequeños orificios de la superficie y provocar daños físicos de mayor envergadura. Otros agentes, como organismos y microorganismos también pueden actuar causando deterioros de carácter físico junto con alteraciones químicas en la superficie del vidrio, como consecuencia de la excreción de determinados ácidos.

Los procesos de degradación anteriormente descritos también se pueden acelerar a causa de las técnicas de fabricación y elaboración de los objetos. En algunos casos, 
una adición insuficiente de determinados componentes provoca la disgregación del material, como se ha observado en los primeros vidrios elaborados con natrón (Freestone 2001: 617). Otros, como la presencia de infundidos y burbujas, generados por los sistemas de elaboración, una fusión deficiente de la mezcla vitrificable y un mal afinado, causan zonas susceptibles de fracturas y agrietamientos tras un impacto. Si estas anomalías o defectos se sitúan cerca de la superficie del vidrio se producen grietas con facilidad y una pérdida de material, formando pequeñas cavidades donde la acumulación de humedad desarrolla otro tipo de alteraciones de carácter químico. Este fenómeno también afecta a las irregularidades o improntas en la superficie que, en el caso de los primeros vidrios de ventana, pueden acelerar la degradación en uno de sus lados por la presencia de huellas derivadas del proceso de elaboración.

Es, sin embargo, en el momento de su descubrimiento cuando los materiales experimentan un cambio brusco de aclimatación después de un largo y lento proceso de adaptación y equilibrio con el medio, por lo que los tratamientos aplicados tras su recuperación son decisivos para su conservación. Generalmente, muchos de los vidrios expuestos en los museos se encuentran desprovistos de las capas de degradación ya que, según algunos investigadores, obstaculizan la legibilidad original de los mismos (Koob 2006). Otros autores argumentan que pueden propiciar nuevos ataques químicos debido a su elevada higroscopicidad (Barrio 2003). Actualmente se considera necesaria su conservación para evitar la exposición del vidrio original a nuevas alteraciones $y$, en el caso del vidrio de ventana, estas capas mantienen algunas huellas y restos imprescindibles para identificar las técnicas de elaboración de los paneles y sus sistemas de instalación.

Para conocer cómo afectan todos los agentes de alteración anteriormente mencionados a una tipología de vidrio poco investigada en la Península lbérica como es el vidrio de ventana romano, se seleccionó un grupo de fragmentos entre varios yacimientos situados dentro de la delimitación de la antigua provincia romana de la Bética. Estos materiales se estudiaron arqueométricamente mediante la observación y microanálisis de sus superficies y de la masa del vidrio, utilizando técnicas adecuadas para ello como microscopía electrónica de barrido de emisión de campo (MEBEC) y espectrometría de dispersión de energías de rayos $X$ (EDS). Los objetivos de este trabajo son: (a) conocer los procesos de degradación del vidrio de ventana romano de la Bética; (b) identificar las posibles causas de estas alteraciones; $y$ (c) determinar posibles actuaciones que atenúen un deterioro acelerado y una pérdida de información de las capas superficiales determinante para reconstruir los patrones tecnológicos de esta clase de objetos.

\section{Materiales: un conjunto de vidrios de ventana romanos procedentes de la Bética}

Para la ejecución del presente estudio se seleccionó un grupo de 15 fragmentos de vidrio de ventana romano procedentes de varios yacimientos de la Bética: Torreparedones (Baena, Córdoba), el conjunto termal de Herrera (Sevilla), Acinipo (Ronda, Málaga), Colonia Patricia Corduba (Córdoba) y Cortalago (Minas de Riotinto, Huelva) [figura $1]$.

Por su morfología todos los fragmentos se corresponden con vidrios de ventana elaborados mediante la técnica de colada y posterior estirado de la masa sobre una superficie. Este sistema fue, probablemente, el utilizado para la obtención de los primeros vidrios empleados para el cierre de vanos en la arquitectura romana (Kisa 1908: 364-365; Allen 2002). Mediante este proceso se obtenían paneles de gran espesor con dos lados bien diferenciados, uno mate rugoso generado por la transferencia en el vidrio de la impronta de la superficie de trabajo y otro brillante liso debido al proceso de enfriamiento de la masa vítrea y su posterior solidificación, de ahí que sean frecuentemente denominados como vidrios de ventana brillante/mate (Boon 1966; Foy y Fontaine 2008: 409). En este último lado es habitual observar algunas de las marcas de las herramientas empleadas en el proceso de estirado, sobre todo en las zonas más externas del panel (Kisa 1908: 365; Boon 1966: 44; Foy y Fontaine 2008: 409). Todos estos rasgos son imprescindibles para determinar la técnica de elaboración y distinguirla de otras posteriores, como las de soplado en cilindros o manchones y soplado en coronas o cibas. Respecto a la masa, son vidrios translúcidos de color natural verde-azulado, una tonalidad debida a la presencia de impurezas de hierro en las materias primas (Price y Cottam 1998: 15).

\section{Metodología, preparación de las muestras y técnicas de análisis}

Para la identificación de los procesos de alteración del conjunto de vidrios de ventana romanos de la Bética se realizó una toma de muestra de cada uno de los fragmentos. Éstos, a su vez, se seleccionaron entre otros ejemplares documentados en los yacimientos tras evaluar el estado de conservación de las capas de degradación de los vidrios, considerando de interés aquéllos que habían sido sometidos claramente a un proceso de limpieza y otros que no.

De cada una de las muestras tomadas se obtuvo una sección mediante corte con disco de diamante. Estas secciones fueron posteriormente embutidas en una resina polimérica en probetas de $3 \mathrm{~cm}$ de diámetro y pulidas a espejo con óxido de cerio en suspensión. Una vez obtenidas las probetas se recubrieron con carbono como medio conductor (vaporizadas en un equipo JEOL JEE4b).

Cada uno de los vidrios se observó con microscopía electrónica de barrido de emisión de campo (MEBEC) y se microanalizó con espectrometría de dispersión 


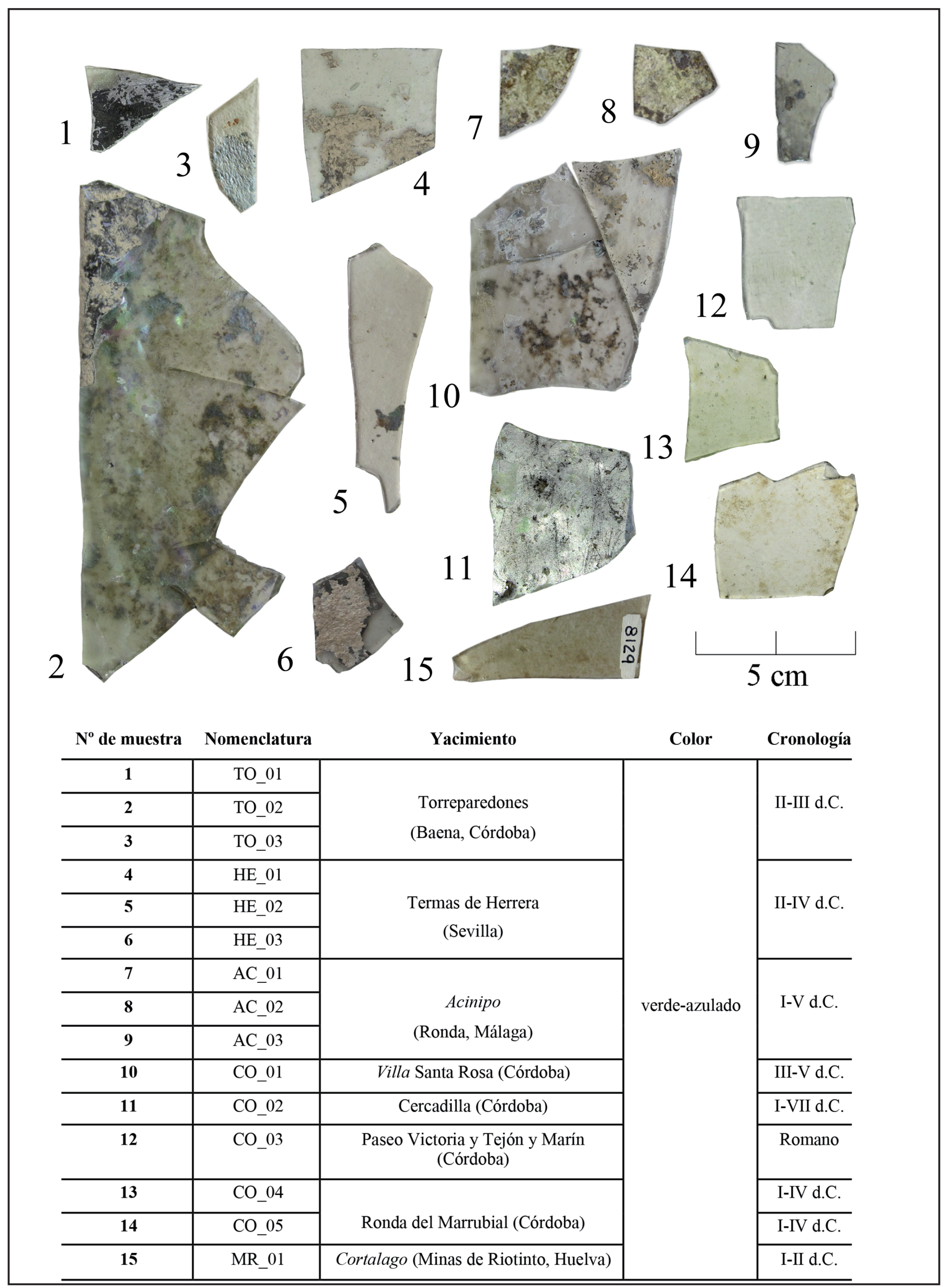

Figura 1.- Muestras de vidrio de ventana romano procedente de varios yacimientos de la Bética seleccionadas para su observación mediante MEBEC y microanálisis EDS. 
de energías de rayos $X$ (EDS). Para ello se utilizó un microscopio de cátodo frío Hitachi modelo S-4800, trabajando con tensiones de aceleración de $15 \mathrm{kV}$. En algunas de las zonas observadas se realizaron microanálisis mediante espectrometría de dispersión de energías de rayos $X$ (EDS) con un espectrómetro acoplado al microscopio, modelo Oxford X-Max de 20 $\mathrm{mm}^{2}$ con resolución de $125 \mathrm{eV}(\mathrm{Mn} \mathrm{Ka})$. Los resultados semicuantitativos de los microanálisis EDS se obtuvieron mediante el sistema de corrección ZAF y se expresaron en $\%$ en peso en óxidos para los siguientes elementos: sodio $(\mathrm{Na})$, magnesio $(\mathrm{Mg})$, aluminio $(\mathrm{Al})$, silicio $(\mathrm{Si})$, fósforo $(\mathrm{P})$, cloro $\left(\mathrm{Cl}^{-}\right)$, potasio $(\mathrm{K})$, calcio $(\mathrm{Ca})$, manganeso $(\mathrm{Mn})$ y hierro (Fe). Las concentraciones de cada uno de los óxidos se corresponden con un área seleccionada en la muestra.

\section{Resultados obtenidos y discusión}

En el examen macroscópico de las superficies se pudo apreciar que todos los casos estudiados mostraban el fenómeno de iridiscencia. No obstante, las capas de degradación del vidrio no se habían conservado de la misma manera en el conjunto debido, posiblemente, a diferencias en los tratamientos que recibieron los materiales tras su descubrimiento. La observación microscópica de las muestras confirmó este primer análisis visual. De los 15 fragmentos analizados, solo dos de ellos (muestras 1 y 11) conservaban las características capas de degradación de un vidrio enterrado. Una parte importante del conjunto (muestras 2, 4, 6, 7, 8, 9, 10, 13 y 14) presentó restos parciales de dichas capas, mientras que las cuatro muestras restantes (muestras 3, 5, 12 y 15) carecían de ellas.

En las muestras donde fue posible visualizar las capas de degradación (muestras 1 y 11), los espesores alcanzaban entre 60 y $140 \mu \mathrm{m}$. En concreto, la capa de gel de sílice tenía aproximadamente $140 \mu \mathrm{m}$ en su lado brillante en la muestra 1, mientras que en la muestra 11 en torno a 100 $\mu \mathrm{m}$. En ambos casos estas medidas eran muy inferiores a las del vidrio conservado, debido en gran parte al espesor que presentan los vidrios de ventana romanos elaborados por colada. Esta característica, junto con la estabilidad química de su composición ha garantizado la conservación de la gran mayoría de las piezas. Los microanálisis EDS en las zonas exteriores indicaron una reducción considerable del contenido de óxido de sodio $\left(\mathrm{Na}_{2} \mathrm{O}\right)$ y de calcio $(\mathrm{CaO})$ respecto al cuerpo del vidrio en la muestra 1 [figura 2, análisis A1-A3] ( 0,9 y 4,1 \% en peso, respectivamente, en la capa más externa, frente a 15,5 y $7,5 \%$ en peso, respectivamente, en el cuerpo del vidrio). Por el contrario, se produjo un enriquecimiento relativo de los óxidos de silicio $\left(\mathrm{SiO}_{2}\right)$, de aluminio $\left(\mathrm{Al}_{2} \mathrm{O}_{3}\right)$ y de hierro $\left(\mathrm{Fe}_{2} \mathrm{O}_{3}\right) 82,5 ; 9,1$ y $0,4 \%$ en peso, respectivamente, en la capa más externa, frente a 71,$2 ; 2,1$ y $0,1 \%$ en peso, respectivamente, en el cuerpo del vidrio. Los datos mostraban, por tanto, un vidrio bastante alterado y con un grado elevado de desalcalinización a nivel superficial, en el que se había formado una "capa de gel de sílice". La muestra 11 proporcionó, en su lado brillante, unos datos muy similares a los obtenidos en la muestra 1 [figura 2, análisis B1-B3].

\begin{tabular}{|ccccccccccccc|}
\hline & & & \\
\hline
\end{tabular}

Figura 2.- Imágenes de MEBEC de las capas de degradación del lado brillante de los vidrios de las muestras (A) 1 (Torreparedones) y (B) 11 (Cercadilla); y del lado mate de la muestra (C) 11 (Cercadilla). Se adjunta una tabla con los resultados de los microanálisis EDS (\% en peso) realizados en varias zonas de cada una de las muestras (--- no determinado). 
Estos dos vidrios también conservaban las capas de degradación en el lado mate. En el caso de la muestra 11 alcanzaban alrededor de $60 \mu \mathrm{m}$ de espesor y en ellas se apreciaron algunos depósitos de suciedad y tierra procedentes de los contextos de enterramiento. Los microanálisis realizados en estas zonas mostraron, al igual que en el lado brillante, un vidrio alterado con bastante desalcalinización superficial, como indicaban los bajos porcentajes de óxido de sodio (3,6 \% en peso) y el enriquecimiento relativo de los óxidos de silicio $(78,9 \%$ en peso), de aluminio (11,7\% en peso) y de hierro $(1,0 \%$ en peso) [figura 2, análisis C1-C4].

Las muestras donde las capas de degradación se habían conservado de manera parcial (muestras 2, 4, 6, 7, 8, 9, 10, 13 y 14), exhibían una pequeña lámina más o menos uniforme [figura 3]. Estas áreas mostraban una composición química similar a los ejemplos anteriormente mencionados, con un contenido inferior de óxidos de sodio $\left(\mathrm{Na}_{2} \mathrm{O}\right)$ y de calcio $(\mathrm{CaO})$ y un incremento de óxido de silicio $\left(\mathrm{SiO}_{2}\right)$ y de óxido de aluminio $\left(\mathrm{Al}_{2} \mathrm{O}_{3}\right)$ [figura 3, análisis $\mathrm{A} 1-\mathrm{F} 2$ ]. En las muestras 6 y 10 los microanálisis EDS efectuados en algunas zonas muy superficiales [figura 3, análisis C2 y E2] revelaron, además, un elevado porcentaje de óxido de calcio $(27,8$ y $71,4 \%$ en peso, respectivamente) por la presencia de costras carbonatadas, una patología que visualmente se detectaba en casi toda la superficie del fragmento perteneciente a la muestra 6 [figura 1.6]. Aunque la capa de degradación debía conservarse bajo esta costra, la manipulación para la toma de muestras propició su desprendimiento en zonas periféricas. Los depósitos calcáreos, también compuestos por otros óxidos (p. ej. $\mathrm{SiO}_{2}, \mathrm{Al}_{2} \mathrm{O}_{3}$ y $\mathrm{Fe}_{2} \mathrm{O}_{3}$ ), procedían del

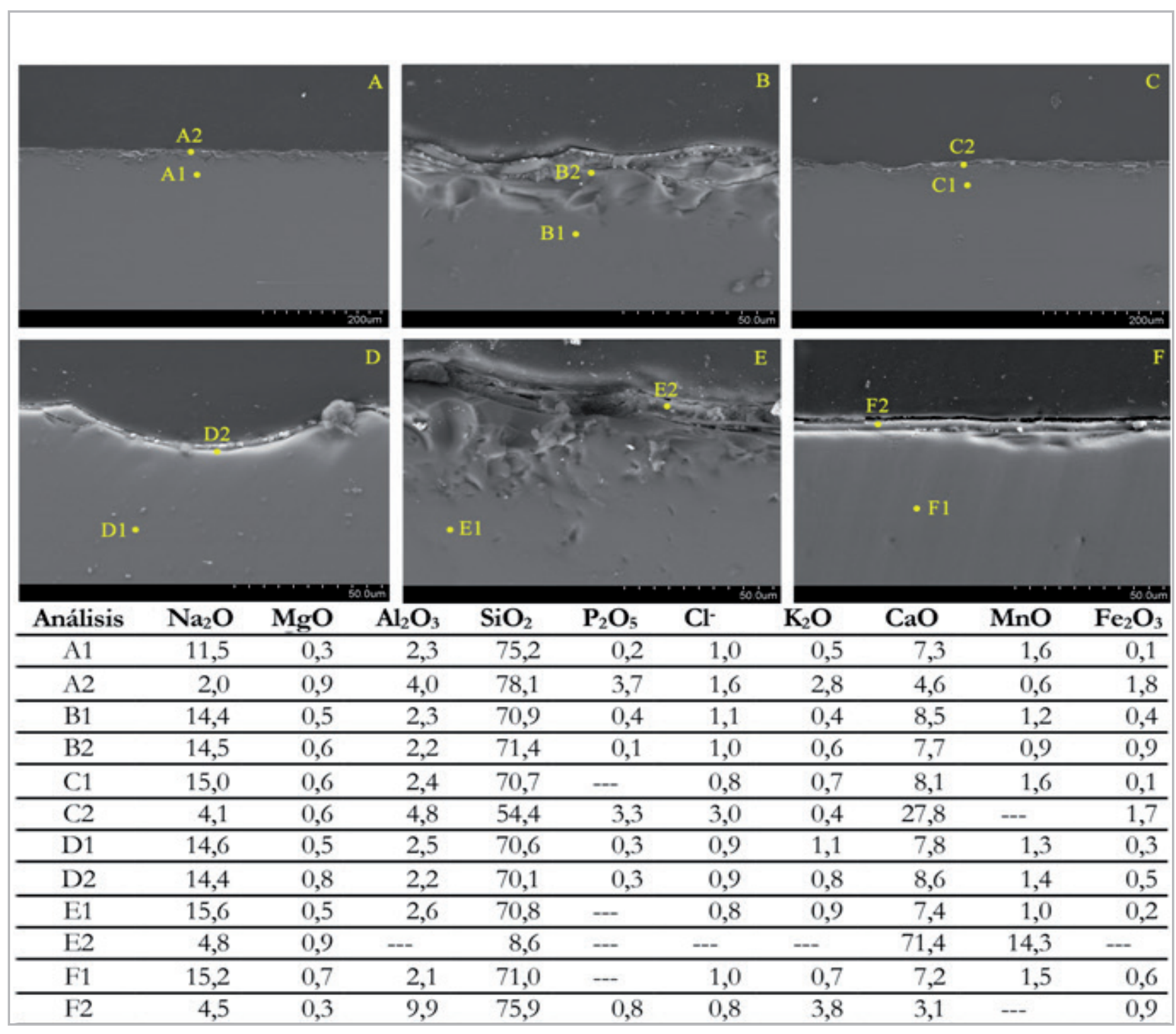

Figura 3.- Imágenes de MEBEC de las capas de degradación parcialmente conservadas de los vidrios: (A) lado mate de la muestra 2 (Torreparedones, Baena); (B) lado mate la muestra 4 (Termas de Herrera); (C) lado brillante de la muestra 6 (Termas de Herrera); (D) lado mate de la muestra 7 (Acinipo, Ronda); (E) lado brillante de la muestra 10 (villa de Santa Rosa, Córdoba); (F) y lado mate de la muestra 13 (villa de Ronda del Marrubial, Córdoba). Se adjunta una tabla con los resultados de los microanálisis EDS (\% en peso) realizados en varias zonas de cada una de las muestras (--- no determinado). 


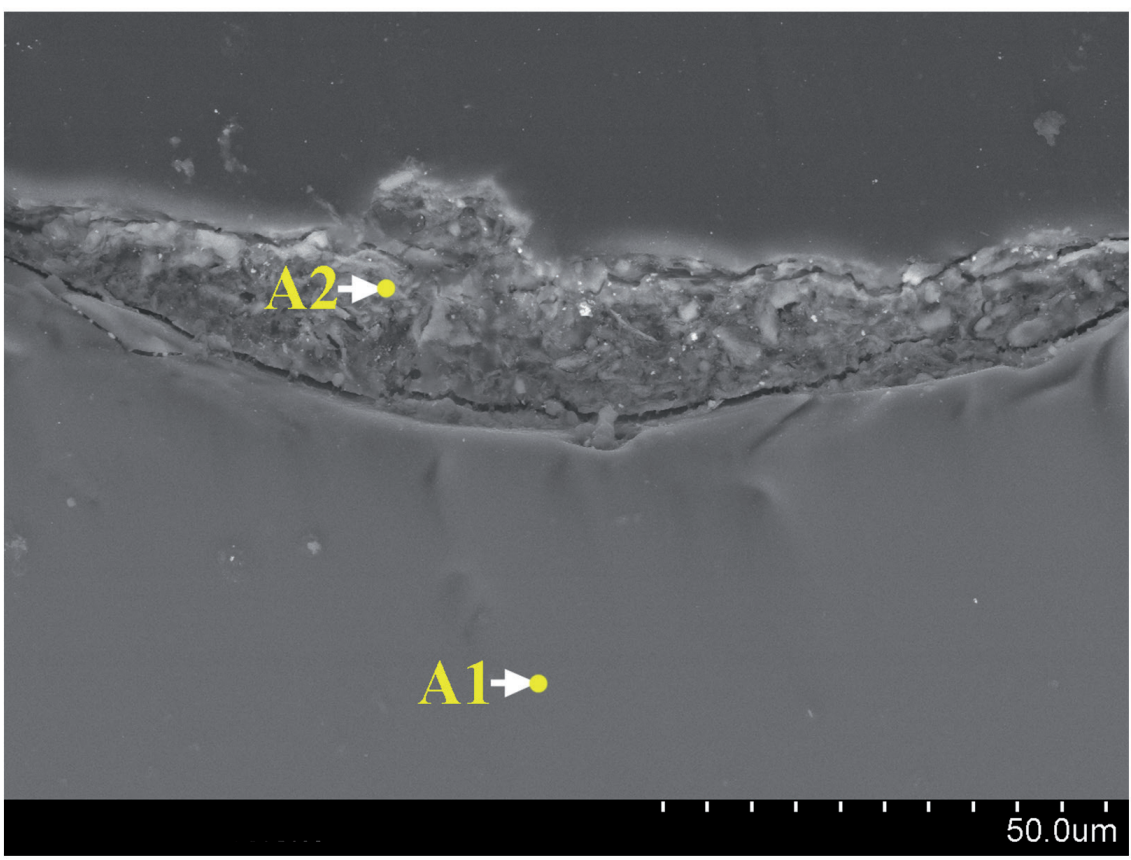

\begin{tabular}{crrrrrrrrrr} 
Análisis & $\mathrm{Na}_{2} \mathrm{O}$ & \multicolumn{1}{c}{$\mathrm{MgO}$} & $\mathrm{Al}_{2} \mathrm{O}_{3}$ & $\mathrm{SiO}_{2}$ & $\mathrm{P}_{2} \mathrm{O}_{5}$ & $\mathbf{C l}^{-}$ & $\mathrm{K}_{2} \mathrm{O}$ & $\mathrm{CaO}_{2}$ & $\mathbf{M n O}$ & $\mathrm{Fe}_{2} \mathrm{O}_{3}$ \\
\hline $\mathrm{A} 1$ & 14,8 & 0,6 & 2,5 & 71,1 & --- & 0,8 & 1,2 & 7,6 & 1,3 & 0,1 \\
\hline $\mathrm{A} 2$ & 1,0 & 2,8 & 15,2 & 44,7 & 2,2 & 0,4 & 2,4 & 25,1 & 0,8 & 5,4 \\
\hline
\end{tabular}

Figura 4.- Imagen de MEBEC de los depósitos carbonatados en la superficie brillante de un fragmento de vidrio procedente del yacimiento de Acinipo (Ronda, Málaga) (muestra 8). Se adjunta una tabla con los resultados de los microanálisis EDS (\% en peso) realizados en varias zonas de la muestra (--- no determinado).

sedimento en el que los vidrios permanecieron enterrados, en estos casos un sustrato de areniscas calcáreas. Carbonataciones de mayor espesor también se visualizaron en algunas irregularidades de otros fragmentos analizados, como en la muestra 8 ( $25,1 \%$ en peso) [figura 4 , análisis A2]. La principal diferencia con los restos de mortero o masilla utilizados para la instalación de los paneles es que estos últimos forman depósitos menos homogéneos, situados en las zonas de los bordes de los paneles y tienen un color más blanquecino respecto a las sales insolubles (Velo Gala y Merino Aranda 2017).

En cambio, en cuatro de las muestras analizadas (muestras $3,5,12$ y 15) no se detectaron las capas de degradación a nivel macroscópico y tras su observación mediante MEBEC [figura 5].

La inexistencia de las capas de degradación en la mayoría de los fragmentos se puede atribuir, principalmente, a dos factores. Por un lado, es posible que se hubiese producido un desprendimiento de las mismas a consecuencia de un cambio brusco de las condiciones ambientales en las que se encontraban los vidrios. Por otro lado, puede atribuirse a los tratamientos de manipulación, almacenaje y limpieza mecánico-manuales, estos últimos a veces muy agresivos, a los que suelen someterse los materiales arqueológicos en las excavaciones. Esta ausencia no solo constituye un riesgo para el desarrollo de nuevos procesos de degradación en la superficie original del vidrio, sino que conlleva una pérdida total o parcial de las huellas que definen las técnicas de elaboración de estos paneles. Estos tratamientos también pueden favorecer el desprendimiento de restos conservados en la superficie, como los morteros o masillas de sellado utilizados en los sistemas de instalación de los paneles, o marcas de óxidos que pueden señalar el uso de bastidores metálicos para su sujeción. El examen macroscópico permitió observar en la superficie mate de algunos fragmentos, que sí conservaron estas capas de degradación, pequeñas líneas u orificios generados por la propia naturaleza del soporte en el que se elaboraron por colada los paneles, o por la aplicación de partículas de arena para evitar la adhesión de la masa del vidrio sobre el plano de trabajo [figura 6A]. En cambio, en aquellos donde el vidrio original había quedado al descubierto, pudo apreciarse claramente una disminución de las improntas [figura 6A-B]. A nivel microscópico estas diferencias son menos perceptibles, aunque sí se distinguieron una serie de micro-fracturas e irregularidades en la parte externa por posibles impactos o abrasiones que constituyen puntos de ataque preferencial para el desarrollo de procesos de corrosión puntuales [figura 7]. 


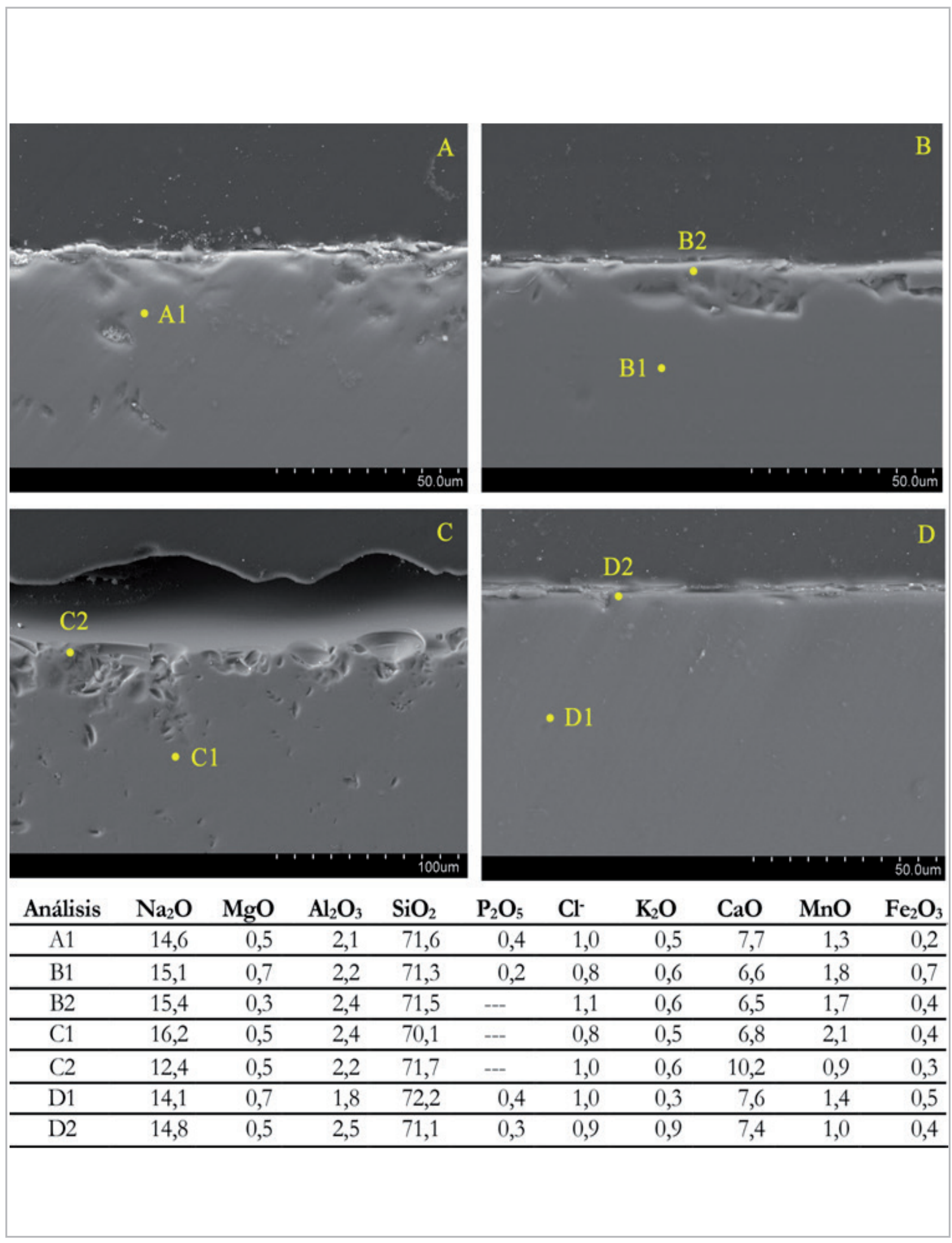

Figura 5.- Imágenes de MEBEC de los vidrios carentes de las capas de degradación: (A) lado brillante de la muestra 3 (Torreparedones, Baena); (B) lado mate la muestra 5 (Termas de Herrera); (C) lado mate de la muestra 12 (Paseo de la Victoria y Tejón y Marín, Córdoba); (D) lado mate de la muestra 15 (Cortalago, Minas de Riotinto). Se adjunta una tabla con los resultados de los microanálisis EDS (\% en peso) realizados en varias zonas de cada una de las muestras (--- no determinado). 


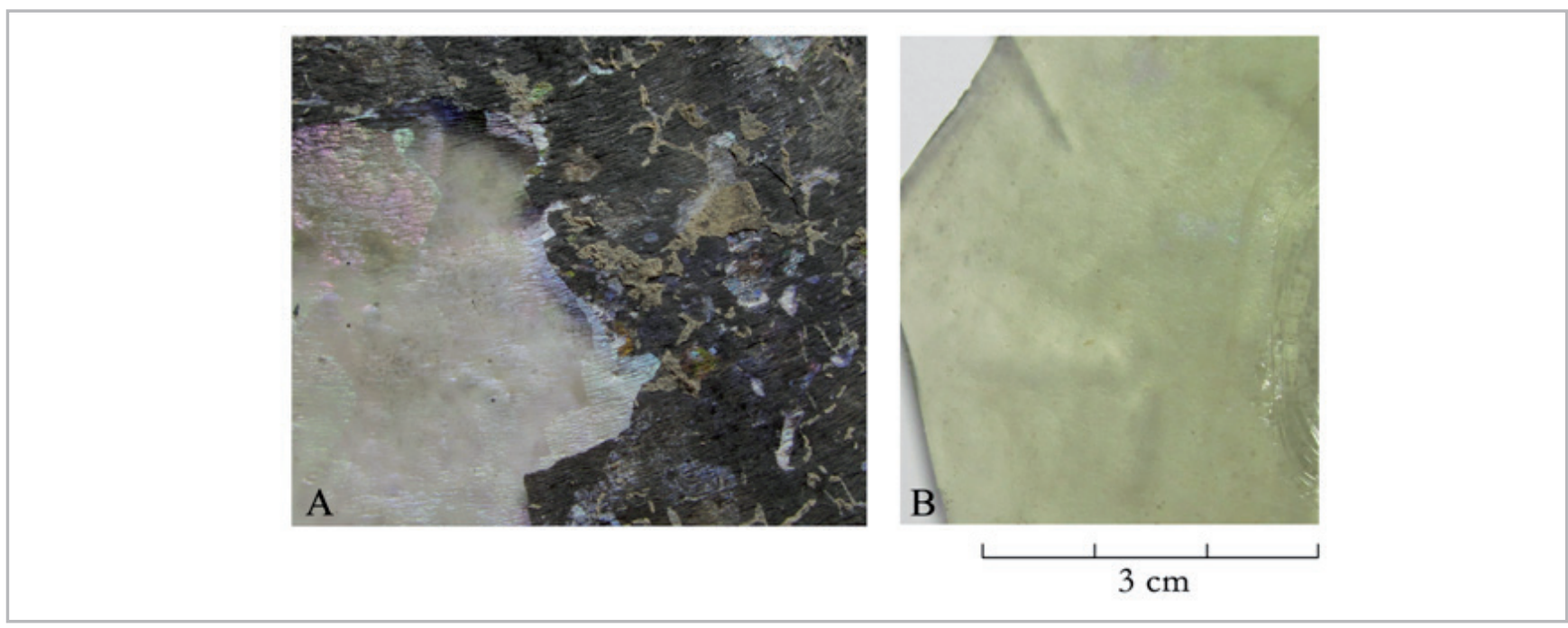

Figura 6.- Imágenes de las improntas de las superficies mate de fragmentos de vidrio de ventana romano: (A) fragmento no sometido a tratamientos de limpieza (Torreparedones, Baena); (B) vidrio en el que se ha aplicado la limpieza de las superficies eliminando las capas de degradación (villa de Ronda del Marrubial, Córdoba).

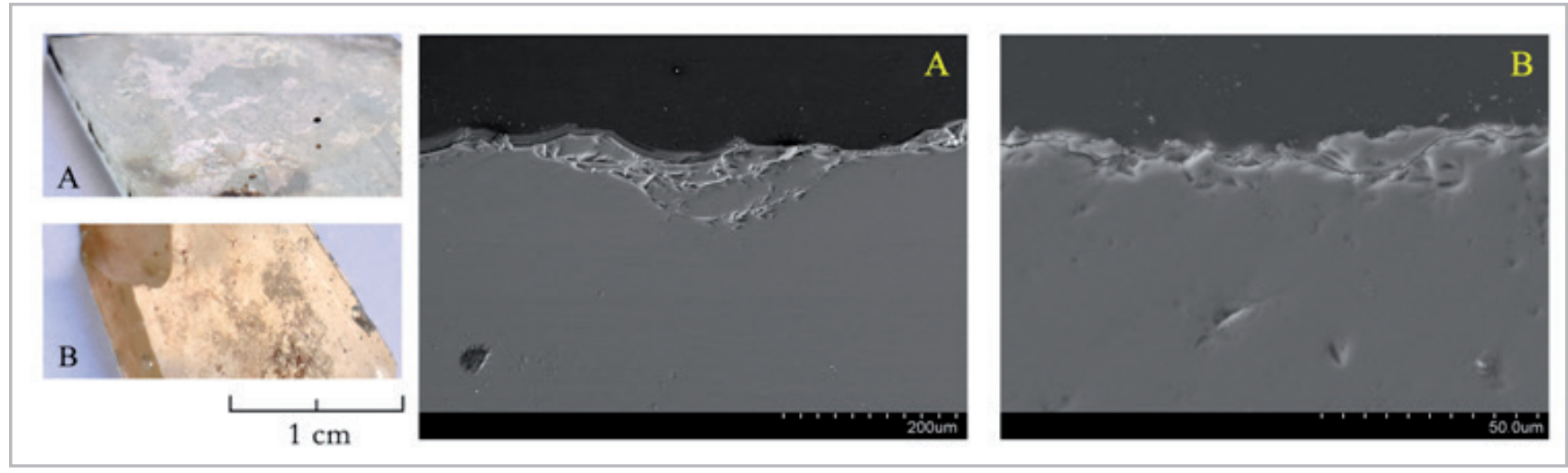

Figura 7.- Imágenes de la superficie y micrografías MEBEC que muestran los daños en el lado brillante de algunos vidrios de ventana romanos de la Bética generados, posiblemente, por impactos entre fragmentos almacenados en un mismo recipiente o por los tratamientos de limpieza mecánico-manual: (A) muestra 9 (Acinipo, Ronda) y (B) 15 (Cortalago, Minas de Riotinto).

En relación con los procesos de alteración físicos, algunas de estas patologías, como pequeñas grietas o fisuras, desencadenaron un deterioro químico en zonas más internas del vidrio, como se apreció en la muestra 1 [figura 8]. El fragmento presentaba una serie de grietas, denominadas por su forma de tipo pluma ${ }^{[1]}$, que posiblemente se originaron por presiones durante las fases de abandono del edificio en el que estos paneles estuvieron instalados o por los contextos de enterramiento. La concentración de la humedad en estas zonas provocó que la degradación afectase a una parte interna del vidrio. La disposición de las láminas de la capa de degradación superficial sobre aquellas formadas en torno a la fisura indicaba, además, que estas últimas se generaron en una fase previa. El escaso intercambio de agua en estos espacios estrechos favoreció un aumento del pH y un avance más acelerado del deterioro en estas zonas respecto a otras áreas externas. Los microanálisis EDS efectuados en la capa de degradación de una de las grietas indicaron una gran disminución de óxido de sodio $\left(\mathrm{Na}_{2} \mathrm{O}\right)$ y el relativo aumento de los óxidos de silicio $\left(\mathrm{SiO}_{2}\right)$ y de aluminio $\left(\mathrm{Al}_{2} \mathrm{O}_{3}\right)$, similar al de aquellas áreas exteriores del vidrio [figura 9, análisis A1-A2]. En la parte central de la fisura se distinguieron unas láminas delgadas perpendiculares a la superficie cuya composición química [figura 9, análisis A3] difería de las anteriores. Todos los microanálisis efectuados en esta zona se caracterizaron por la elevada concentración de óxidos de manganeso ( $\mathrm{MnO}: 34,9 \%$ en peso) y de hierro $\left(\mathrm{Fe}_{2} \mathrm{O}_{3}: 10,1 \%\right.$ en peso). El aumento en el porcentaje de ambos componentes puede explicarse por: una migración de estos óxidos desde el interior del vidrio, un proceso conocido en vidrios romanos (Schalm et al. 2011); por el aporte externo de hierro y manganeso, elementos comunes en la composición de los suelos, que tras penetrar en solución a través de estas pequeñas grietas cristalizaron (Cox y Ford 1993: 5646; Silvestri et al. 2005: 1346); o por un efecto sinérgico de estos dos procesos. 


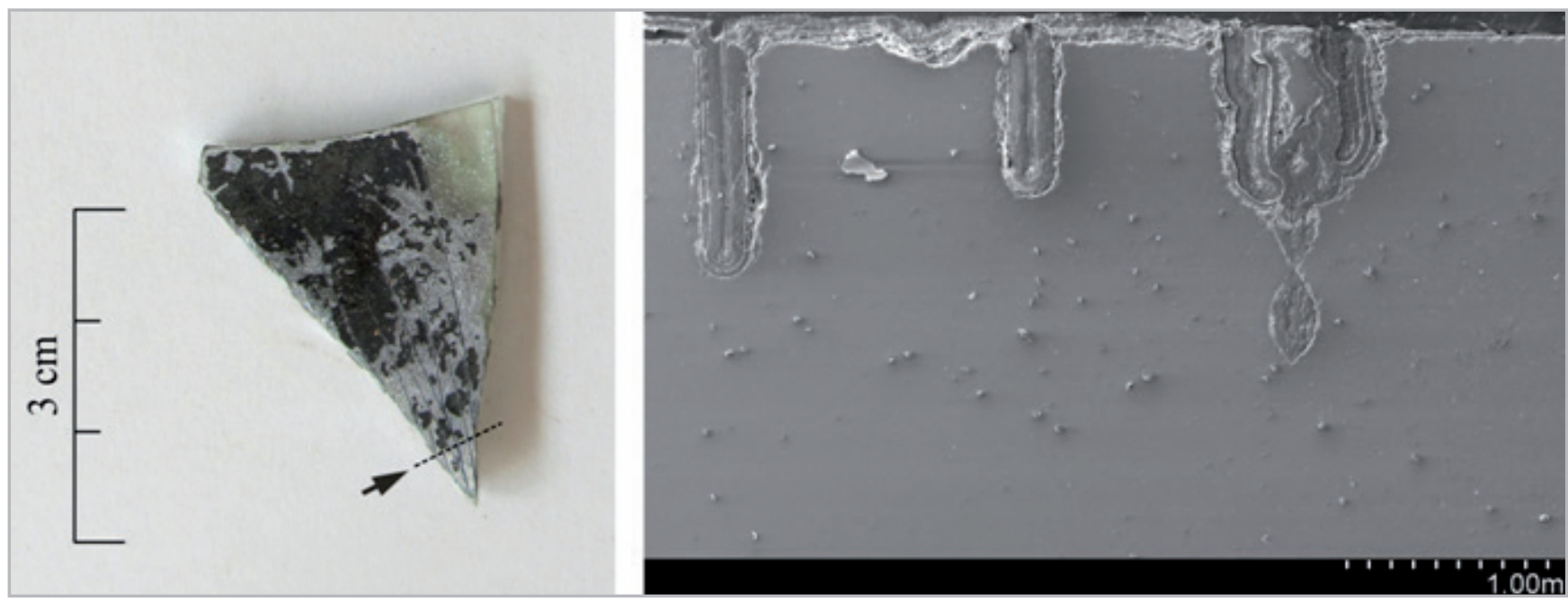

Figura 8.- Fotografía del fragmento de vidrio de ventana romano del yacimiento de Torreparedones (Baena) en donde se indica la sección analizada (izq.) y micrografía MEBEC de la muestra 1 en sección (dcha.).

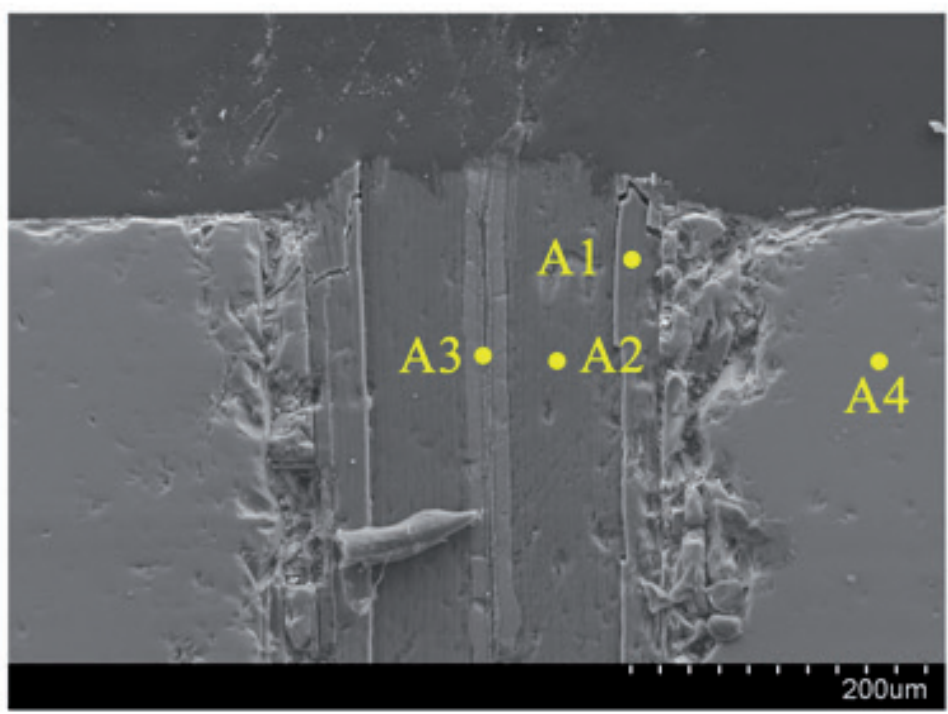

\begin{tabular}{crrrrrrrrrr} 
Análisis & $\mathrm{Na}_{2} \mathbf{O}$ & $\mathbf{M g O}$ & $\mathrm{Al}_{2} \mathbf{O}_{3}$ & $\mathbf{S i O}_{2}$ & $\mathbf{P}_{2} \mathbf{O}_{5}$ & $\mathbf{C l}^{-}$ & $\mathbf{K}_{2} \mathbf{O}$ & $\mathbf{C a O}$ & $\mathbf{M n O}$ & $\mathrm{Fe}_{2} \mathbf{O}_{3}$ \\
\hline $\mathrm{A} 1$ & 1,0 & 0,7 & 7,5 & 85,5 & 0,1 & 0,1 & 1,8 & 3,2 & $\cdots$ & 0,1 \\
\hline $\mathrm{A} 2$ & 1,0 & 1,2 & 8,6 & 80,2 & --- & 0,5 & 1,9 & 5,9 & 0,5 & 0,2 \\
\hline $\mathrm{A} 3$ & 0,8 & 0,8 & 4,5 & 38,1 & 0,9 & 0,7 & 1,0 & 8,0 & 34,9 & 10,1 \\
\hline $\mathrm{A} 4$ & 14,96 & 0,69 & 2,29 & 70,56 & 0,17 & 0,72 & 0,44 & 8,18 & 1,48 & 0,48 \\
\hline
\end{tabular}

Figura 9.- Imagen de MEBEC de las capas de degradación en una fisura de tipo pluma de un fragmento de vidrio de ventana romano procedente del yacimiento de Torreparedones (Baena, Córdoba) (muestra 1). Se adjunta una tabla con los resultados de los microanálisis EDS (\% en peso) realizados en varias zonas de la muestra (--- no determinado).

Las observaciones mediante MEBEC han permitido, además, el examen de las características del cuerpo del vidrio de los fragmentos estudiados. De manera general, todas las muestras analizadas revelan una masa de gran homogeneidad. Sin embargo, existen algunos infundidos y numerosas burbujas de aire causados por una fusión deficiente de la mezcla de vidrio, un mal afinado o su manipulación para obtener los paneles, que pueden facilitar los procesos de alteración en estos objetos. Este es el caso de una de las muestras analizadas en sección (muestra 6), en la que se observó una burbuja de aire próxima a la superficie del vidrio, que podría llegar a desencadenar procesos de degradación más agresivos por acumulación de humedad en estas zonas [figura 10 A]. Por último, en el cuerpo del vidrio de la muestra 14 se apreció una microestructura compuesta por una serie 

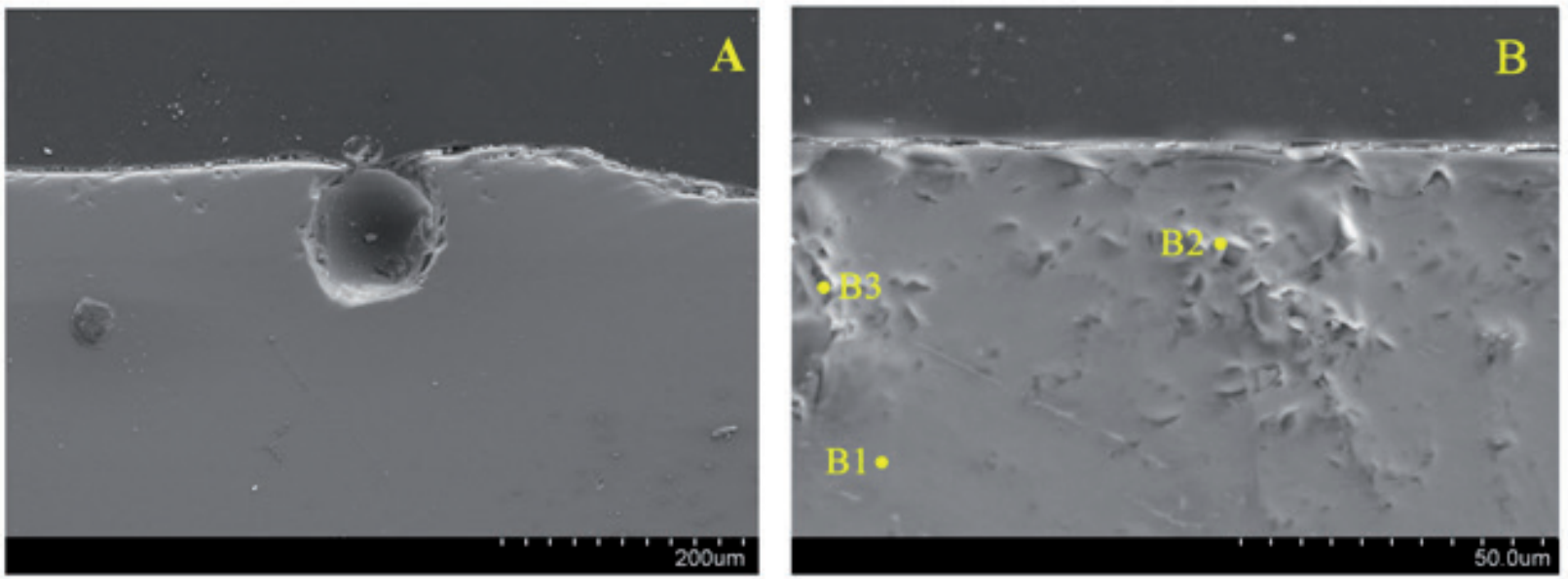

\begin{tabular}{crrrrrrrrrr} 
Análisis & $\mathrm{Na}_{2} \mathrm{O}$ & $\mathbf{M g O}$ & $\mathrm{Al}_{2} \mathrm{O}_{3}$ & $\mathrm{SiO}_{2}$ & $\mathrm{P}_{2} \mathrm{O}_{5}$ & $\mathrm{Cl}^{-}$ & $\mathrm{K}_{2} \mathrm{O}$ & $\mathbf{C a O}$ & $\mathbf{M n O}$ & $\mathrm{Fe}_{2} \mathrm{O}_{3}$ \\
\hline $\mathrm{B} 1$ & 14,3 & 0,8 & 1,9 & 72,2 & 0,1 & 1,0 & 0,5 & 7,1 & 1,6 & 0,5 \\
\hline B2 & 13,0 & 0,5 & 1,8 & 71,8 & --- & 1,4 & 0,5 & 8,7 & 1,8 & 0,5 \\
\hline B3 & 11,6 & 0,6 & 2,1 & 74,1 & --- & 0,9 & 0,4 & 8,0 & 1,7 & 0,6 \\
\hline
\end{tabular}

Figura 10.- (A) Imagen de MEBEC de una burbuja de aire en la masa del vidrio de uno de los fragmentos procedente de las termas de Herrera, Sevilla (muestra 6); (B) Imagen de MEBEC de la microestructura presente en la masa del vidrio de uno de los fragmentos de la villa de Ronda del Marrubial (Córdoba) (muestra 14). Se adjunta una tabla con los resultados de los microanálisis EDS (\% en peso) realizados en varias zonas de la muestra 14 (--- no determinado).

de partículas de aspecto cristalino distribuidas de manera generalizada por toda la sección del vidrio observada [figura $10 \mathrm{~B}$ ]. Los resultados de los análisis realizados en varias zonas revelaron una composición química muy similar a la de un vidrio de silicato sódico cálcico de base natrón ( $\mathrm{MgO}$ y $\mathrm{K}_{2} \mathrm{O}$ por debajo de 1,0 \% en peso), en el que probablemente se utilizó dióxido de manganeso como agente decolorante ( $\mathrm{MnO}$ entre 1,6 y 1,8\% en peso) [figura 10, análisis B1-B3]. Estos resultados indican que no se trata de procesos de desvitrificación del vidrio (Fernández Navarro 2003). La transparencia de los paneles y la ausencia de opacificantes, cuyo aspecto físico se asemeja a las formaciones detectadas (Kaplan et al. 2017), podría indicar que estas formaciones estén relacionadas con la fina capa conductora de grafito debido a un aumento de grano medio de la película en el proceso de vaporización (Rahman et al. 2005: 278, figura 3).

\section{Conclusiones}

El estudio arqueométrico del estado de conservación de un grupo de 15 fragmentos de vidrio de ventana procedentes de varios yacimientos de época romana situados en la Bética ha proporcionado datos importantes sobre los procesos de degradación de esta tipología de vidrio, los tratamientos de limpieza y los sistemas de almacenamiento aplicados a estos materiales tras su hallazgo.

Todos los fragmentos presentan un estado de conservación aceptable debido a que son vidrios de silicato sódico cálcico con composiciones características de época romana. Estas composiciones, de gran estabilidad química, el espesor de los fragmentos y las condiciones ambientales de los contextos de enterramiento, han garantizado una buena preservación de una parte importante del material original, donde solo las capas superficiales se han visto afectadas por los procesos de degradación del vidrio arqueológico. No obstante, casi todos ellos, a excepción de dos muestras pertenecientes a los yacimientos de Torreparedones y Cercadilla, muestran una pérdida parcial o completa de las capas de alteración. Los microanálisis efectuados en los fragmentos que sí las conservaban, revelaron un enriquecimiento en estas capas de la concentración de óxidos de silicio $\left(\mathrm{SiO}_{2}\right)$, de aluminio $\left(\mathrm{Al}_{2} \mathrm{O}_{3}\right)$ y de hierro $\left(\mathrm{Fe}_{2} \mathrm{O}\right)$ y una reducción de óxidos de sodio $\left(\mathrm{Na}_{2} \mathrm{O}\right)$ y de calcio $(\mathrm{CaO})$, respecto al cuerpo original del vidrio. La ausencia de estas capas en el resto de las muestras se debe, o bien a los tratamientos de limpieza aplicados a estos materiales o bien a un incorrecto almacenaje de los fragmentos, lo que habría provocado su desprendimiento. La observación mediante MEBEC indicó que, incluso, algunos de estos procedimientos habían alterado la superficie del vidrio a nivel microscópico, a consecuencia de impactos o una retirada forzada de la suciedad y las capas de degradación.

El escaso cuidado en el mantenimiento de las capas de degradación en esta clase de vidrios conlleva su pérdida, un aspecto relevante para el estudio de las superficies de estos materiales puesto que, al tratarse de una transformación del vidrio original, reproducen su superficie de manera detallada. La gran mayoría de los 
paneles conserva en estas capas la impronta de las huellas de las herramientas utilizadas en su elaboración y de las superficies de trabajo, lo que resulta imprescindible para definir la técnica de elaboración, en este caso por colado y estirado de la masa de vidrio. Además, algunos de los fragmentos pertenecientes a los bordes conservan restos en superficie relacionados con los morteros o masillas de sellado, como sucede en algunos de los ejemplos recuperados en el yacimiento de Torreparedones. Si se procede a la eliminación indiscriminada de estas capas, estos restos desaparecen de las superficies, junto con la suciedad y las costras carbonatadas a veces confundidos con esta clase de depósitos, impidiendo conocer de esta forma cómo se instalaron los paneles.

Los resultados de este estudio conducen a plantear la necesidad de aplicar una serie de pautas de actuación en los vidrios de ventana romanos desde el momento de su hallazgo hasta su posterior almacenamiento. Antes de acometer un proceso de limpieza en estos materiales es preciso realizar labores de preconsolidación de estas capas, incluso en el momento de su extracción si éstas se encuentran en peligro de desprendimiento. Asimismo, es imprescindible documentar de manera exhaustiva la pieza o fragmento, identificando todas las huellas y depósitos superficiales, garantizando la consolidación de aquellos que permitan obtener una información destacada. En lo que respecta al almacenaje de estos materiales se considera que debe realizarse en las condiciones idóneas para evitar un avance de los procesos de degradación en la superficie del vidrio (humedad relativa entre 40 y $45 \%$ ), depositando cada fragmento de manera individual en un recipiente que evite colisiones o movimientos bruscos.

\section{Notas}

[1] Las fisuras del tipo "pluma" son formaciones causadas por un agrietado espontáneo debido a daños físicos en la superficie del vidrio, y se caracterizan por generar una grieta a partir de la cual surgen otras de menor tamaño dispuestas paralelamente y en un ángulo de unos $45^{\circ}$ respecto a la central (Davison 2003: 184).

\section{Agradecimientos}

Los autores agradecen la financiación del proyecto Retos de la Sociedad del Ministerio de Ciencia e Innovación (Ref. PID2019104220RB-100) y del programa TOP Heritage de la Comunidad de Madrid y fondos estructurales de la Unión Europea (Ref. S2018/NMT-4372). También el apoyo profesional de la Red TechnoHeritage de Ciencia y Tecnología para la Conservación del Patrimonio Cultural y de la Plataforma Temática Interdisciplinar del CSIC Patrimonio Abierto: Investigación y Sociedad (PTIPAIS). También expresan su agradecimiento a Fernando Agua Martínez, responsable técnico del Laboratorio de Conservación del Patrimonio del Instituto de Historia (CSIC), por su ayuda en la preparación de las muestras.

\section{Referencias}

AGUA MARTÍNEZ, F.; CONDE MORENO, J.F.; OÑATE BAZTÁN, P.; SANGUINOVÁZQUEZ, J.; DÁVILA SERRANO, A.; GARCÍA-HERAS, M.; VILLEGAS BRONCANO, M.A. (2015). “Caracterización y estado de conservación de vidrios tardorromanos del Museo Arqueológico Regional de Madrid procedentes de las necrópolis de Cubas de la Sagra", Boletín de la Sociedad Española de Cerámica y Vidrio, 54 (2): 58-68. https://doi.org/10.1016/j.bsecv.2015.03.003

ALLEN, D. (2002). "Roman window glass". En Artefacts and Archaeology. Aspects of the Celtic and Roman world, AldhouseGreen, M.; Webster, P. (eds.). Cardiff: University of Wales Press, 102-111.

BARRIO, J. (2003). "Evaluación crítica de los principios en Arqueometría, Conservación y Restauración de los vidrios arqueológicos", Pátina: Revista de la Escuela de Conservación y Restauración de Bienes Culturales, 12: 53-64.

BOON, G.C. (1966). "Roman window glass from Wales”, Journal of Glass Studies, 8: 41-45. https://www.jstor.org/stable/24184877

COX, G.A.; FORD, B.A. (1993). "The long-term corrosion of glass by ground-water", Journal of Materials Science, 28: 5637-5647. https://doi.org/10.1007/BF00367840

DAVISON, S. (2003). Conservation and restoration of glass, $2^{\mathrm{a}}$ edición. Oxford: Butterworth-Heinemann.

FERNÁNDEZ NAVARRO, J.M. (2003). El vidrio. Constitución, fabricación, propiedades. Madrid: Consejo Superior de Investigaciones Científicas, 3a edición.

FREESTONE, I.C. (2001). "Post depositional changes in archaeological ceramics and glass". En Handbook of Archaeological Sciences, Brothwell, D.R.; Pollard, A.M. (eds.). Chichester: John Wiley \& Sons Ltd, 615-625.

FOY, D.; FONTAINE, S.D. (2008). “Diversité et évolution du vitrage de l'Antiquité et du Haut Moyen Âge", Gallia, 65: 405-459. https:// doi.org/10.3406/galia.2008.3349

GARCÍA-HERAS, M.; VILLEGAS BRONCANO, M.A. (2004). "Notas para el estudio científico del vidrio antiguo", Zephyrus, 57: 377390. https://revistas.usal.es/index.php/0514-7336/article/ view/5414

KAPLAN, Z.; IPEKOGLU, B.; BOKE, H. (2017). "Physicochemical properties of glass tesserae in Roma terrace house from ancient Antandros (base glass, opacifiers and colorants), Mediterranean Archaeology and Archaeometry, 17(1): 141-157. https://doi. org/10.5281/zenodo. 258103

KISA, A. (1908). Das glas im Altertume, 2. Leipzig: Verlag Von Karl W. Hiersemann.

KOOB, S.P. (2006). Conservation and care of glass objects. LondresNueva York: Archetype Publications \& Corning Museum of Glass. 
PALOMAR SANZ, T.; GARCÍA-HERAS, M.; SABIO GONZÁLEZ, R.; RINCÓN LÓPEZ, J.M.; VILLEGAS BRONCANO, M.A. (2012). "Composition, preservation and production technology of Augusta Emerita Roman glasses from the first to the sixth century AD", Mediterranean Archaeology \& Archaeometry, 12 (2): 193-211. http://www.maajournal.com/

PALOMAR SANZ, T.; OUIJA, M.; CASTILLEJO STRIANO, M.; SABIO GONZÁLEZ, R.; RINCÓN LÓPEZ, J.M.; GARCÍA-HERAS, M.; VILLEGAS BRONCANO, M.A. (2013). "Roman glasses from Augusta Emerita: study of the degradation pathologies using LIBS". En Science and technology for the conservation of Cultural Heritage, RogerioCandelera, M.A.; Lazzari, M.; Cano Díaz, E. (eds.). Londres: A. A. Balkema Publishers, Taylor \& Francis Group, 251-255. http://www. routledge.com/books/details/9781138000094/

PRICE, J.; COTTAM, S. (1998). Romano-British glass vessels: a handbook. York: Council for British Archaeology.

RAHMAN, M.Y.A.; SALLEH, M.M.; TALIB, I.A.; YAHAYA, M. (2005): "Solid state photoelectrochemical cells utilising graphite thin fils counter electrode", Ionics, 11(3):275-280. https://doi.org/10.1007/ BF02430389

SCHALM, O.; PROOST, K.; DE VIS, K.; CAGNO, S.; JANSSENS, K.; MEES, F.; JACOBS, P.; CAEN, J. (2011). "Manganeses staining of archaeological glass: the characterization of $\mathrm{Mn}$-rich inclusions in leached layers and a hypothesis of its formation", Archaeometry, 53(1): 103-112. https://doi.org/10.1111/j.1475-4754.2010.00534.x

SILVESTRI, A.; GIANMARIO, M.; SALVIULO, G. (2005). “Archaeological glass alteration products in marine and land-based environments: morphological, chemical and microtextural characterization", Journal of Non-Crystalline Solids, 351: 1338-1349. https://doi. org/10.1016/j.jnoncrysol.2005.03.013

VELO GALA, A.; MERINO ARANDA, A. (2017). "La curia de Torreparedones: un nuevo modelo de restitución a partir del estudio de otros materiales", Actas del Décimo Congreso Nacional y Segundo Congreso Internacional Hispanoamericano de Historia de la Construcción. Donostia-San Sebastián, 3-7 de octubre 2017, 1707-1716.

VELO-GALA, A.; GARCÍA-HERAS, M.; ORFILA, M. (2019). “Roman window glass in Hispania Baetica: glass origin and manufacture study through Electrone Microprobe analysis", Journal of Archaeological Science: Reports, 24: 526-538. https://doi. org/10.1016/j.jasrep.2019.01.021

VELO-GALA, A. (2020): "El reciclaje del vidrio en época romana". En Reutilización y reciclaje. Reflexiones desde la Arqueología, Velo Gala, A. Sánchez López, E. Orfila Pons, M. (eds.). Granada: Editorial Universidad de Granada, 89-106.

\section{Autor/es}

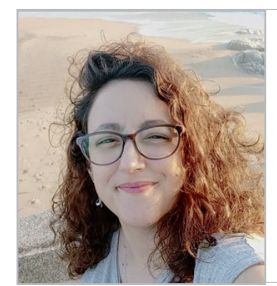

\section{Almudena Velo-Gala}

almudenya@correo.ugr.es

Universidad de Granada

Licenciada en Bellas Artes y especializada en Restauración del patrimonio arqueológico y pictórico por la Universidad de Granada. Es doctora en Historia y Artes (2019) por esta misma Universidad, donde realizó su tesis titulada 'El vidrio de ventana y su empleo en la arquitectura romana de la Bética'. Desde el año 2011 ha dedicado sus investigaciones a la conservación y arqueología del vidrio, especialmente de los periodos romano y andalusí. Es colaboradora del equipo Post-disciplinary and Experimental Glass Group de la Universidad de Newcastle (UK), con quien ha participado en proyectos en dos sitios declarados Patrimonio Mundial de la UNESCO: Madinat al-Zahra y la Alhambra.

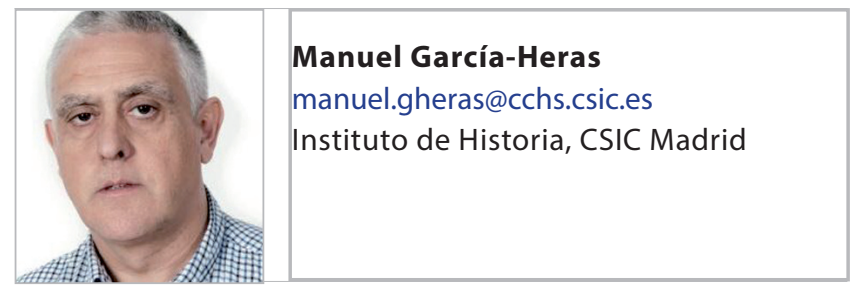

Doctor en Historia con Premio Extraordinario por la Universidad Complutense de Madrid (1997). Entre 1999 y 2001 fue investigador postdoctoral Fulbright en la Smithsonian Institution, Washington D.C., EE.UU.; y entre 2002 y 2005 investigador postdoctoral del programa I3P en el Centro Nacional de Investigaciones Metalúrgicas (CSIC, Madrid). Desde 2005 es Científico Titular en el Instituto de Historia (CSIC, Madrid). Sus líneas de investigación se centran en la interacción entre ciencias experimentales e historia, combinando una visión histórica y científica en la aproximación a cuestiones de tecnología y conservación de materiales antiguos. Estas líneas se llevan a cabo principalmente en los campos de la Arqueometría y la conservación del Patrimonio Cultural.

Artículo enviado el 11/01/2021 Artículo aceptado el 24/03/2021

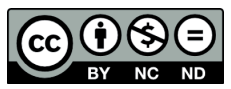

https://doi.org/10.37558/gec.v19i.880 